INTERSTITIAL LUNG DISEASE

\title{
Short course dexamethasone treatment following injury inhibits bleomycin induced fibrosis in rats
}

\author{
W A Dik, R J McAnulty, M A Versnel, B A E Naber, L J I Zimmermann, G J Laurent, \\ S E Mutsaers*
}

Thorax 2003;58:765-771

See end of article for authors' affiliations

\section{Correspondence to:}

Dr W A Dik, Department of Immunology, Erasmus MC, University Medical Centre Rotterdam, P O Box 1738 3000 DR Rotterdam, The Netherlands;

w.dik@erasmusmc.nl

Revised version received 5 March 2003

Accepted for publication

3 April 2003

\begin{abstract}
Background: Corticosteroids are routinely used in patients with pulmonary fibrosis. The timing for initiation of treatment is likely to be crucial for corticosteroids to exert an antifibrotic effect. Experimental studies in animals have examined the effect of corticosteroid treatment starting before or at the time of lung injury. However, this is not representative of the human condition as treatment only begins after disease has been established. We examined the effect of a short course corticosteroid treatment starting 3 days after bleomycin induced lung injury on the development of pulmonary fibrosis.

Methods: Bleomycin $(1.5 \mathrm{mg} / \mathrm{kg})$ was instilled intratracheally into rats to induce pulmonary fibrosis. The effect of a 3-day course of dexamethasone $(0.5 \mathrm{mg} / \mathrm{kg})$ initiated 3 days after bleomycin induced lung injury on cell proliferation and collagen deposition was examined by analysing bronchoalveolar lavage (BAL) fluid and lung tissue.

Results: Treating bleomycin exposed animals after injury with dexamethasone for 3 days inhibited lung collagen deposition compared with animals exposed to bleomycin without dexamethasone treatment (15.2 (2.2) mg collagen/lung $v 22.5$ (2.1) mg/lung; $p<0.05)$. Dexamethasone treatment reduced pulmonary parenchymal cell proliferation in bleomycin exposed rats but did not influence BAL fluid mitogenic activity for lung fibroblasts or alter the BAL fluid levels of the fibrogenic mediators transforming growth factor- $\beta_{1}$, platelet derived growth factor- $A B$, and thrombin.

Conclusions: A 3 day course of dexamethasone treatment initiated 3 days after bleomycin induced lung injury reduces lung cell proliferation and collagen deposition by mechanisms other than through reduction of transforming growth factor- $\beta_{1}$, platelet derived growth factor- $A B$, and thrombin levels in BAL fluid. We propose that an early short course treatment with dexamethasone may be useful in inhibiting pulmonary fibrosis.
\end{abstract}

$\mathrm{P}$ ulmonary fibrosis is the end stage of a heterogeneous group of disorders of known and unknown aetiology. Despite the wide variety of insults associated with this condition-such as bacterial infection, inhalation of organic and inorganic dusts, radiation, drugs, and trauma-the mechanisms involved appear largely the same. ${ }^{1}$ It is assumed that, in response to injury, inflammatory cells enter the lung and, together with resident lung cells, release mediators that stimulate fibroblast proliferation and collagen deposition within the lung interstitium. In addition to this, mediators influencing fibroblast proliferative activity may also originate from the blood. 'A host of mediators has been implicated in the pathogenesis of pulmonary fibrosis because they fit three basic criteria: (1) they stimulate fibroblast replication or procollagen synthesis; (2) the gene expression and protein production of the mediator is increased in the lungs of patients with pulmonary fibrosis; and (3) inhibitors of the mediator function attenuate fibrosis in animal models of the disease. $^{2}$ Mediators which fit these criteria include platelet derived growth factor (PDGF), transforming growth factor- $\beta_{1}$ (TGF- $\left.\beta_{1}\right)$, insulin like growth factor-1, endothelin-1, fibronectin, and thrombin. ${ }^{13-9}$

The classical pathogenetic model for acute respiratory distress syndrome (ARDS) suggests that epithelial and endothelial damage results in an inflammatory phase that is followed by a fibroproliferative phase which, if excessive,

*Current address: Asthma and Allergy Research Institute and Department of Medicine, University of Western Australia, Nedlands, Western Australia 6009 results in established fibrosis. The current hypothesis, however, is that fibroproliferation occurs in parallel with inflammation. ${ }^{10}$ We have recently shown that fibroproliferation is indeed an early response to lung injury in ARDS and may therefore be an important therapeutic target. ${ }^{11}$ Treatment of ARDS with immunosuppressive corticosteroids was reported to be more effective in inhibiting fibrosis development when administered early in the fibroproliferative phase-that is, before dense acellular fibrosis with deranged alveolar architecture occurs. This response to treatment was found to be associated with pulmonary improvement and a decrease in the $\mathrm{N}$-terminal propeptides of collagen types I and III in plasma and bronchoalveolar lavage (BAL) fluid. ${ }^{12}{ }^{13}$ These findings clearly show that the time point at which corticosteroid treatment is initiated is crucial for exerting its antifibrotic effect.

Neonatal respiratory distress syndrome has pathophysiological features comparable to ARDS and can progress towards bronchopulmonary dysplasia (BPD). The progression towards BPD is associated with the development of fibrosis. ${ }^{14}$ Interestingly, short course treatment with the corticosteroid dexamethasone started 12-48 hours after birth in infants with neonatal respiratory distress syndrome increased survival without BPD and reduced the requirement for subsequent late dexamethasone treatment. ${ }^{15}{ }^{16}$ This suggests that such a short course of corticosteroid treatment initiated early after injury is able to prevent lung fibrosis, but this has not been studied directly. If short early treatment with dexamethasone indeed prevents the development of fibrosis, it may be of clinical importance in the treatment of patients at risk of developing pulmonary fibrosis. 
Intratracheal instillation of the antitumour agent bleomycin is the most commonly used animal model for pulmonary fibrosis. This model is characterised by an early predominantly neutrophilic inflammatory response, increased fibroblast proliferation, and enhanced collagen deposition due to increased collagen synthesis and decreased collagen degradation. ${ }^{17}{ }^{18}$ In addition, it has been shown that fibroproliferative activity coexists with inflammation, and that the major proliferative phase occurs during the first week after bleomycin induced injury. ${ }^{19}{ }^{20}$ Previous studies using prolonged administration of corticosteroids such as methylprednisolone or dexamethasone initiated before or simultaneously with bleomycin reduced pulmonary inflammation, lung injury, and collagen deposition in this model. ${ }^{21-23}$ However, as anti-inflammatory treatment usually starts after lung injury has occurred, the clinical relevance of starting treatment in animals before or at the time of injury is questionable.

A study was undertaken to determine whether a 3 day course of dexamethasone treatment $(0.5 \mathrm{mg} / \mathrm{kg}$ body weight $)$ initiated 3 days after the induction of lung injury with bleomycin affected collagen deposition and cell proliferation and modulated the expression profile of the profibrotic mediators TGF- $\beta_{1}$, PDGF-AB, and thrombin in BAL fluid.

\section{METHODS}

\section{Corticosteroid treatment in the bleomycin model}

Male Lewis rats were intratracheally injected with bleomycin disulphate $(1.5 \mathrm{mg} / \mathrm{kg}$ body weight in $0.3 \mathrm{ml}$ saline; Kyowa Hakko, Slough, UK) as described previously. ${ }^{6}$ After 3 days the animals were given a daily intraperitoneal injection of dexamethasone sodium phosphate $(0.5 \mathrm{mg} / \mathrm{kg}$ body weight in $0.5 \mathrm{ml}$ saline; Sigma, St Louis, MO, USA) or saline for 3 days. Groups of six rats were killed 3, 7, and 14 days after bleomycin or saline instillation and their lungs were lavaged three times with $4 \mathrm{ml}$ saline. Total and differential cell counts were performed and the BAL fluid was aliquoted and stored at $-80^{\circ} \mathrm{C}$. The lungs were removed, blotted dry, and snap frozen in liquid nitrogen. The lung collagen content and BAL fluid mitogenic activity and growth factor levels were measured for all groups.

For examination of cell proliferation, groups of three animals were killed 3 and 5 days after bleomycin or saline injection with and without dexamethasone treatment. Animals were injected intraperitoneally with bromodeoxyuridine (BrdU; $15 \mu \mathrm{g} / \mathrm{g}$ body weight in $0.3 \mathrm{ml}$ saline; Sigma) 1 hour before killing, and the lungs were removed and processed for immunohistochemical analysis as previously described. ${ }^{6}$ Paraffin sections $(5 \mu \mathrm{m})$ were stained using a BrdU Staining Kit (ZYMED Laboratories Inc; South San Francisco, CA, USA) according to the manufacturer's instructions. The number of BrdU positive cells was determined by light microscopy in 10 high power fields of lung tissue (400× magnification per section). Institutional approval was obtained to perform these animal studies.

\section{Collagen measurement}

Frozen powdered lung samples were hydrolysed in $2 \mathrm{ml}$ of $6 \mathrm{M}$ $\mathrm{HCl}$ at $110^{\circ} \mathrm{C}$ for 16 hours and the hydroxyproline content of the proteins was measured. ${ }^{6}$ The amount of collagen in total lung tissue was calculated assuming that lung collagen contains $12.2 \% \mathrm{w} / \mathrm{w}$ hydroxyproline, ${ }^{24}$ and expressed as $\mathrm{mg}$ collagen/lung.

\section{Fibroblast proliferation assay}

Human fetal lung fibroblasts (HFL-1) were seeded at $6 \times 10^{3}$ cells/well into 96-well plates in $50 \mu \mathrm{l}$ Dulbecco's modified Eagle's medium (DMEM; Gibco, Renfrewshire, UK) supplemented with $0.4 \%$ normal calf serum (NCS), L-glutamine, and antibiotics and allowed to adhere for 24 hours. Thereafter, $50 \mu \mathrm{l}$ of a $1 / 4$ dilution of BAL fluid in DMEM/0.4\% NCS was added (six replicates per BAL fluid sample, yielding a 1/8 dilution of BAL fluid) and proliferation assessed after 48 hours using a methylene blue colorimetric assay. ${ }^{25}$

\section{Measurement of growth factors in BAL fluid}

Total protein in BAL fluids was measured using a Bradford based reagent (Bio-Rad; München, Germany). PDGF and total TGF- $\beta_{1}$ (latent and active TGF- $\beta_{1}$ ) were measured using an enzyme linked immunosorbent assay (ELISA; R\&D Systems, Abingdon, UK and Promega; Madison, WI, USA, respectively). Thrombin activity was measured using a colorimetric assay based on the conversion of a thrombin specific substrate (TosGly-Pro-Arg-pNA $(\text { Sigma })^{26}$ ) in the presence of BAL fluid with and without the thrombin inhibitor PPACK (Bachem; Bubendorf, Switzerland) as described previously. ${ }^{27}$

\section{Statistical analyses}

The results are presented as mean (SE) values. Data between groups were compared using the unpaired Student's $t$ test. A p value of $<0.05$ was considered to be statistically significant.

\section{RESULTS}

\section{Animal weights}

Rats injected with bleomycin lost weight during the first 3 days after treatment but gained weight thereafter. Rats treated with bleomycin and receiving dexamethasone continued to lose weight during the 3 days of dexamethasone administration and started to gain weight again when dexamethasone was stopped. Control animals receiving dexamethasone also lost weight during treatment and started to gain weight again when dexamethasone administration was stopped.

\section{BAL fluid total and differential cell counts}

For all animals, recovery of lavage fluid was $83.9(0.7) \%$ of the used lavage volume. Recoveries did not differ between treatment groups or between the examined time points. Table 1 shows the total leucocyte counts and composition of the cell population in BAL fluid from the different groups at the different time points examined. Three days after bleomycin the total number of cells in the BAL fluid was significantly increased compared with the control group. Furthermore, the percentage of neutrophils was significantly higher and the percentage of macrophages significantly lower than in the control animals. At day 14 the total number of cells was still higher in bleomycin treated animals, but the proportion of cell types comprising the BAL cell population was comparable with controls. There was a trend towards decreased cell numbers in the BAL fluid of dexamethasone treated animals compared with controls at days 7 and 14, but these changes were not statistically significant. However, there was a significantly lower percentage of macrophages at days 7 and 14 following bleomycin and dexamethasone treatment compared with control animals, while the percentage of lymphocytes at day 14 was higher than in the control group.

\section{Collagen measurement}

Figure 1 shows the changes in total lung collagen 3, 7 and 14 days after instillation of bleomycin, with and without dexamethasone treatment. Bleomycin had no effect on lung collagen content at day 3. At day 7 the collagen content was significantly increased in the bleomycin group given saline (BLM) compared with the bleomycin group given dexamethasone (BLMdex) (14.2 (1.6) mg/lung $v 9.7$ (0.7) mg/lung; $\mathrm{p}<0.05)$. The collagen content continued to increase in the BLM group and by day 14 was double that of the controls (22.5 (2.1) mg $v 11.2(1.1) \mathrm{mg} ; \mathrm{p}<0.05)$. The lung collagen content in the BLMdex group did not increase compared with control animals treated (CTRLdex) or untreated (CTRL) with dexamethasone at either 7 or 14 days. Animals in the BLMdex 
Table 1 Total and differential BAL fluid cell counts for the four groups of animals at days 3, 7 and 14

\begin{tabular}{|c|c|c|c|c|c|c|c|c|c|c|}
\hline & \multicolumn{2}{|l|}{ Day 3} & \multicolumn{4}{|l|}{ Day 7} & \multicolumn{4}{|l|}{ Day 14} \\
\hline & CTRL & BLM & CTRL & CTRLdex & BLM & BLMdex & CTRL & CTRLdex & BLM & BLMdex \\
\hline $\begin{array}{l}\text { No of cells } / \mathrm{ml} \text { BAL } \\
\text { fluid }\left(\times 10^{4}\right)\end{array}$ & $5.2(1.0)$ & $26.8(5.7)^{*}$ & $10.0(3.0)$ & $5.5(1.3)$ & $10.0(3.8)$ & $7.3(2.8)$ & $5.8(1.3)$ & $4.8(0.6)$ & $10.4(2.0)^{*}$ & $7.2(1.4)$ \\
\hline$\%$ neutrophils & $4.1(2.9)$ & $47.0(5.2)^{*}$ & $1.6(0.7)$ & $1.0(0.4)$ & $24.4(8.6)$ & $15.7(7.4)$ & $0.7(0.3)$ & $1.0(0.2)$ & $2.0(1.0)$ & $5.6(2.1)$ \\
\hline$\%$ macrophages & $95.1(2.9)$ & $46.9(6.8)^{*}$ & $98.3(0.6)$ & $98.3(0.5)$ & $73.2(9.3)$ & $81.1(8.3)^{*}$ & $99.0(0.4)$ & $98.9(0.3)$ & $97.1(1.1)$ & $93.6(2.1)$ * \\
\hline$\%$ lymphocytes & $0.9(0.3)$ & $6.1(1.9)$ & $0.1(0.1)$ & $0.7(0.2)$ & $2.4(0.9)$ & $3.1(1.1)$ & $0.3(0.1)$ & $0.1(0.1)$ & $1.0(0.5)$ & $0.9(0.3)^{*}$ \\
\hline
\end{tabular}

$\mathrm{CTRL}=$ control with saline; $\mathrm{BLM}=$ bleomycin with saline; $\mathrm{CTRLdex=control}$ with dexamethasone; $\mathrm{BLMdex}=$ bleomycin with dexamethasone.

${ }^{*} \mathrm{p}<0.05$ (Student's $t$ test) $v$ appropriate control group.

All groups consisted of six animals except CTRL at day $7(n=3)$ and CTRLdex at day $7(n=5)$.

group had significantly reduced lung collagen at day 14 compared with the BLM group (15.2 (2.2) $\mathrm{mg} v 22.5$ (2.1) $\mathrm{mg}$; $\mathrm{p}<0.05)$. There was no significant change in total lung collagen in control animals at any of the times examined. Treatment of control animals with dexamethasone did not influence the total lung collagen content at any time point.

\section{BAL fluid induced fibroblast proliferation}

Figure 2 shows the mitogenic effect of BAL fluid from animals in each group on fibroblast proliferation over 48 hours. There was a significant increase in BAL fluid induced fibroblast proliferation at day 3 in animals exposed to bleomycin compared with saline treated animals $(28.4(1.8) \%$ proliferation above medium control $v 5.7(1.4) \% ; \mathrm{p}<0.05)$. No differences were observed between bleomycin treated and control animals at days 7 and 14. Treatment with dexamethasone did not significantly influence the mitogenic activity of BAL fluid in bleomycin treated animals at days 7 and 14. At day 7 the BAL fluid from control animals exposed to dexamethasone had significantly less mitogenic activity than bleomycin exposed animals treated with dexamethasone (20.2 (4.9)\% proliferation for BLMdex compared with 3.0 (4.6)\% for CTRLdex; p<0.05). No significant change in BAL fluid mitogenic activity was observed for dexamethasone treated and untreated controls at any of the times examined.

\section{Measurement of growth factors in BAL fluid}

Total protein levels were significantly increased 3 days after instillation of bleomycin (631.1 (56.4) $\mu \mathrm{g} / \mathrm{ml}$ BAL fluid) compared with controls ( 118.8 (47.6) $\mu \mathrm{g} / \mathrm{ml} ; \mathrm{p}<0.05$ ). Thereafter, total protein levels declined in bleomycin treated animals but were still significantly increased compared with controls at

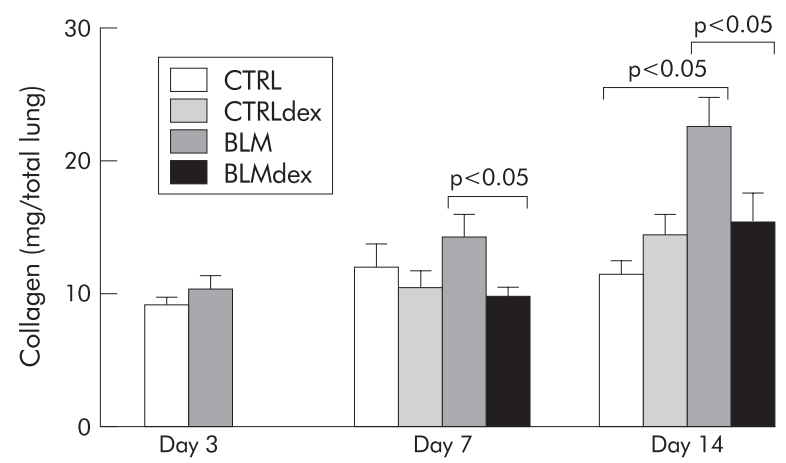

Figure 1 Change in lung collagen content at various times following instillation of bleomycin. The collagen content was measured 3-14 days after intratracheal instillation of bleomycin or saline, with and without a 3 day course of intraperitoneal dexamethasone. Each value represents the mean (SE). Four different treatment groups were studied: (1) bleomycin with saline (BLM); (2) bleomycin with dexamethasone (BLMdex); (3) control with saline (CTRL); and (4) control with dexamethasone (CTRLdex). All groups consisted of six animals except the CTRL group on day $7(n=3)$ and the CTRLdex group on day $7(n=5)$. day $14 \quad(158.5 \quad(26.7) \mu \mathrm{g} / \mathrm{ml} \quad v \quad 78.1 \quad(12.3) \mathrm{ml} ; \quad \mathrm{p}<0.05)$. Treatment with dexamethasone did not influence BAL fluid total protein levels in bleomycin treated or control animals. There was no significant change in BAL fluid total protein levels in controls at any of the times examined (fig 3A).

Total TGF- $\beta_{1}$ levels in BAL fluid were significantly increased at day 3 after bleomycin treatment (616.4 (165.9) pg/ml BAL fluid) compared with controls (98.9 (27.9) pg/ml; $\mathrm{p}<0.05$ ). TGF- $\beta_{1}$ levels declined 7 days after bleomycin but were still higher than in controls (194.6 (48.0) pg/ml $v 46.1$ (12) pg/ml; $\mathrm{p}<0.05)$. At day 14 after bleomycin treatment TGF- $\beta_{1}$ levels were still significantly increased $(230.6(177.7) \mathrm{pg} / \mathrm{ml})$ compared with controls (24.9 (7.6) pg/ml BAL; p<0.05). Treatment of bleomycin exposed and control animals with dexamethasone did not influence TGF- $\beta_{1}$ levels in BAL fluid at any of the times examined. There was no significant change in TGF- $\beta_{1}$ levels in controls at any of the times examined (fig 3B).

PDGF-AB levels in BAL fluid were significantly increased at day 3 after bleomycin $(209.9(25.8) \mathrm{pg} / \mathrm{ml})$ compared with controls (90 (6.2) pg/ml; $\mathrm{p}<0.05)$, and remained raised at relatively constant levels during the study period. Treatment of bleomycin exposed and control animals with dexamethasone did not influence PDGF-AB levels in BAL fluid at any of the times examined. There was no significant change in PDGF-AB levels for controls at any of the times examined (fig 3C).

Thrombin activity in BAL fluid was significantly increased at day 3 after exposure to bleomycin $(0.6(0.07)$ OD units at $405 \mathrm{~nm})$ compared with controls 0.1 (0.03) OD units at $405 \mathrm{~nm} ; \mathrm{p}<0.05)$. Thereafter, thrombin activity gradually declined in BAL fluid of bleomycin treated animals but remained significantly raised at all times examined compared with controls (fig 3D). Treatment of bleomycin exposed and control animals with dexamethasone did not influence thrombin activity in BAL fluid at any of the times examined. In control animals BAL fluid thrombin activity was higher at day 3 than in controls at days 7 and 14 (fig 3D).

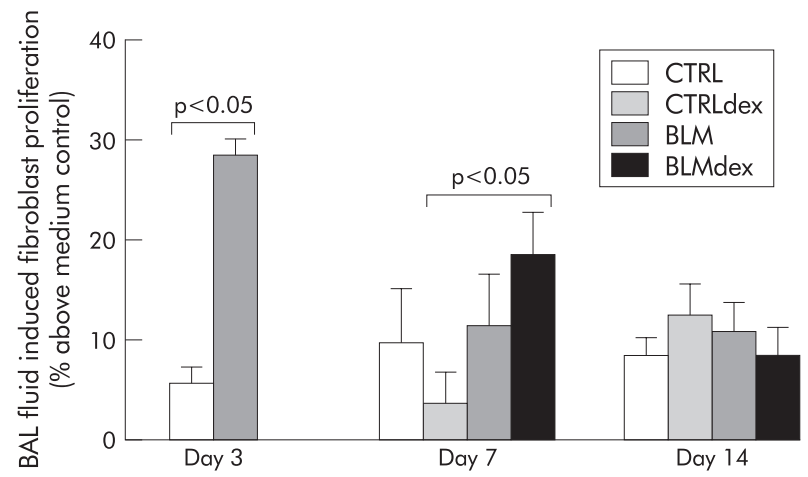

Figure 2 Effect of a $1 / 8$ dilution of BAL fluid on fibroblast proliferation 3-14 days after intratracheal instillation of bleomycin or saline with and without a 3 day course of dexamethasone. Proliferation was expressed as the percentage change in mean absorbance above that for cells exposed to DMEM/0.4\% NCS alone. Each value represents the mean (SE). 

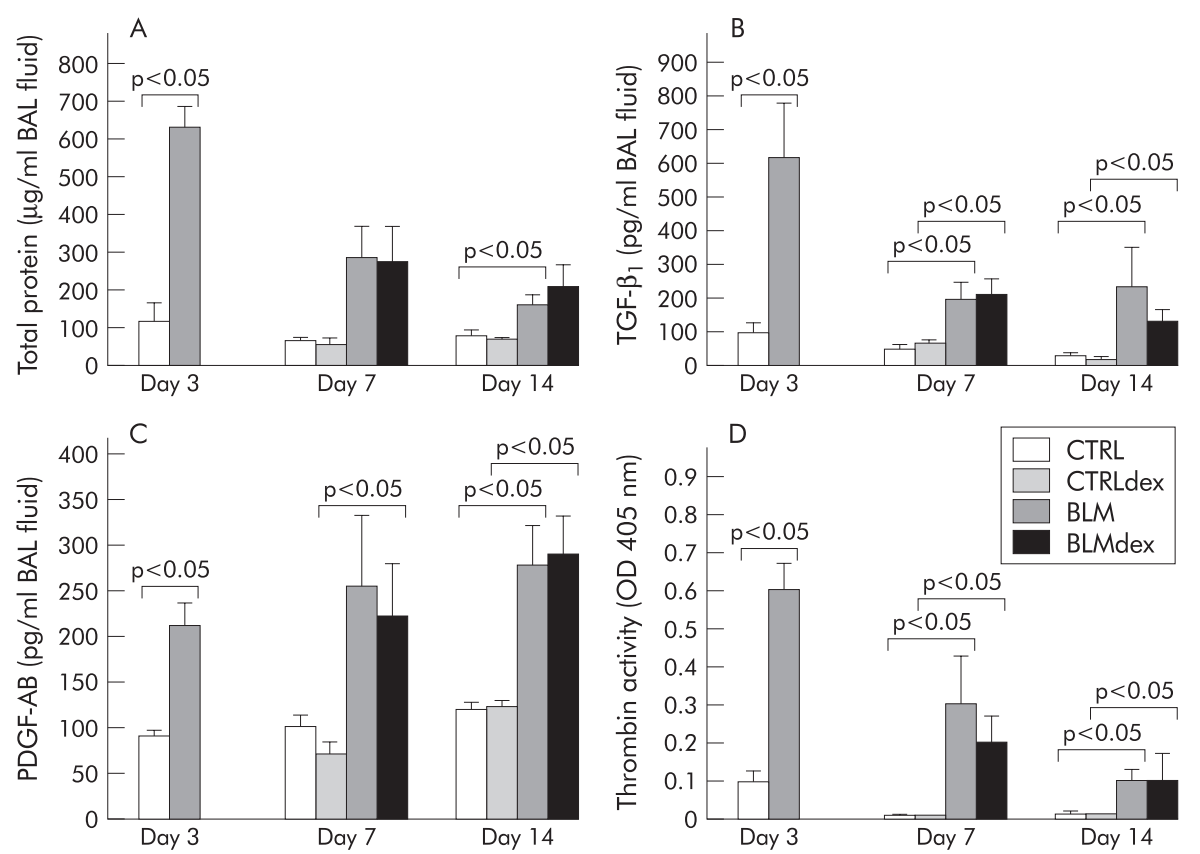

Figure 3 Changes in BAL fluid levels of (A) total protein, (B) total transforming growth factor (TGF)- $\beta_{1}$, (C) platelet derived growth factor (PDGF)-AB, and (D) thrombin 3-14 days after instillation of bleomycin or saline with and without a 3 day course of dexamethasone. The sensitivity of the assays was $25 \mathrm{pg} / \mathrm{ml}$ and $8.4 \mathrm{pg} / \mathrm{ml}$ for TGF- $\beta_{1}$ and PDGF-AB, respectively. Thrombin activity is expressed as optical density at $405 \mathrm{~nm}\left(\mathrm{OD}_{405 \mathrm{~nm}}\right)$, determined as the difference in OD measured at 24 hours between the sample with and without PPACK. Each value represents the mean (SE). Four different treatment groups were studied: (1) bleomycin with saline (BLM); (2) bleomycin with dexamethasone (BLMdex); (3) control with saline (CTRL); and (4) control with dexamethasone (CTRLdex). All groups consisted of six animals except CTRL on day $7(n=3)$ and CTRLdex on day $7(n=5)$

\section{Cell proliferation by BrdU incorporation}

Very few cells were proliferating in the lung tissue of control animals as assessed by BrdU immunoreactivity, with staining limited to a few cells whose appearance and location were consistent with that of alveolar type II epithelial cells (fig 4A). In animals exposed to bleomycin, increased numbers of BrdU positive alveolar type II epithelial resembling cells and interstitial cells were clearly evident both in bleomycin injured areas (fig 4B-D) and in normal areas of lung parenchyma at all times examined. Treatment of bleomycin exposed animals with dexamethasone significantly reduced the number of BrdU positive cells, both in areas of bleomycin induced injury and normal lung parenchyma (fig 5).

\section{DISCUSSION}

Previous studies have shown that prolonged administration of corticosteroids, initiated before or at the same time as bleomycin administration, inhibited the development of lung fibrosis in rats. ${ }^{21-23}$ Although an important finding, its clinical relevance is questionable as anti-inflammatory treatment is always given after lung damage has occurred. In our study the corticosteroid concentration used was much lower than in previous studies, ${ }^{21-23}$ treatment was initiated after bleomycin induced lung injury, and it was only administered for a short time. We clearly show that a 3 day course of dexamethasone treatment $(0.5 \mathrm{mg} / \mathrm{kg} /$ day $)$ started 3 days after bleomycin induced lung injury causes reduced collagen deposition in the lungs of rats 14 days after exposure to bleomycin. From these data we cannot exclude the possibility that dexamethasone delays rather than inhibits the fibrotic response. However, as bleomycin induces acute inflammation, it is unlikely that a further inflammatory response will be seen after 7 days, especially when animals have been treated with dexamethasone. Indeed, the lavage cell counts of animals treated with bleomycin and dexamethasone, although not significantly different, tended to be lower than those in animals treated with bleomycin alone at 7 and 14 days. As there was no increase in collagen deposition compared with controls at day 14 in animals exposed to bleomycin and treated with dexamethasone, it is unlikely that there will be a significant change at later time points, which suggests that our data represent suppression rather than a delay of fibrogenesis.

The current theory on the pathogenesis of pulmonary fibrosis is based on the hypothesis that mediators released by inflammatory cells and resident lung cells as well as blood borne mediators induce fibroblasts to proliferate and/or to produce excess collagen. ${ }^{1}$ In agreement with previous studies, ${ }^{17}{ }^{19}$ we observed a significant increase in the number of inflammatory cells after bleomycin treatment which decreased after 3 days. We observed no significant difference in the inflammatory cell population between dexamethasone treated and untreated bleomycin exposed animals. This may account for the fact that no differences were observed in BAL fluid levels of TGF- $\beta_{1}$ and PDGF-AB between these groups of animals.

TGF- $\beta_{1}$ is a potent stimulator of collagen synthesis by fibroblasts, ${ }^{28}$ and TGF- $\beta_{1}$ mRNA expression is increased before increases occur in type I and type III procollagen mRNAs in bleomycin induced lung fibrosis. ${ }^{29}$ Different approaches to inhibit TGF- $\beta$ have been successfully used to prevent bleomycin induced pulmonary fibrosis. Neutralising TGF- $\beta$ antibodies, soluble TGF- $\beta$ type II receptors, and decorin are all able to inhibit TGF- $\beta_{1}$ activity and reduce collagen accumulation after bleomycin treatment. ${ }^{30-32}$ In our study we detected increased levels of TGF- $\beta_{1}$ in BAL fluid after bleomycin, comparable with levels reported previously. ${ }^{32}{ }^{33}$ Dexamethasone did not downregulate TGF- $\beta_{1}$ levels in BAL fluid at any of the times examined after bleomycin treatment. Although we did not study protein expression by BAL fluid cells, it is likely that alveolar macrophages substantially contributed to the TGF- $\beta_{1}$ levels in BAL fluid as these cells have been shown to express and secrete TGF- $\beta$ after exposure to bleomycin. ${ }^{34}{ }^{35}$ Furthermore, TGF- $\beta_{1}$ levels were reduced seven days after bleomycin treatment, which coincided with a reduction in inflammatory cell numbers. Our observation that dexamethasone was 


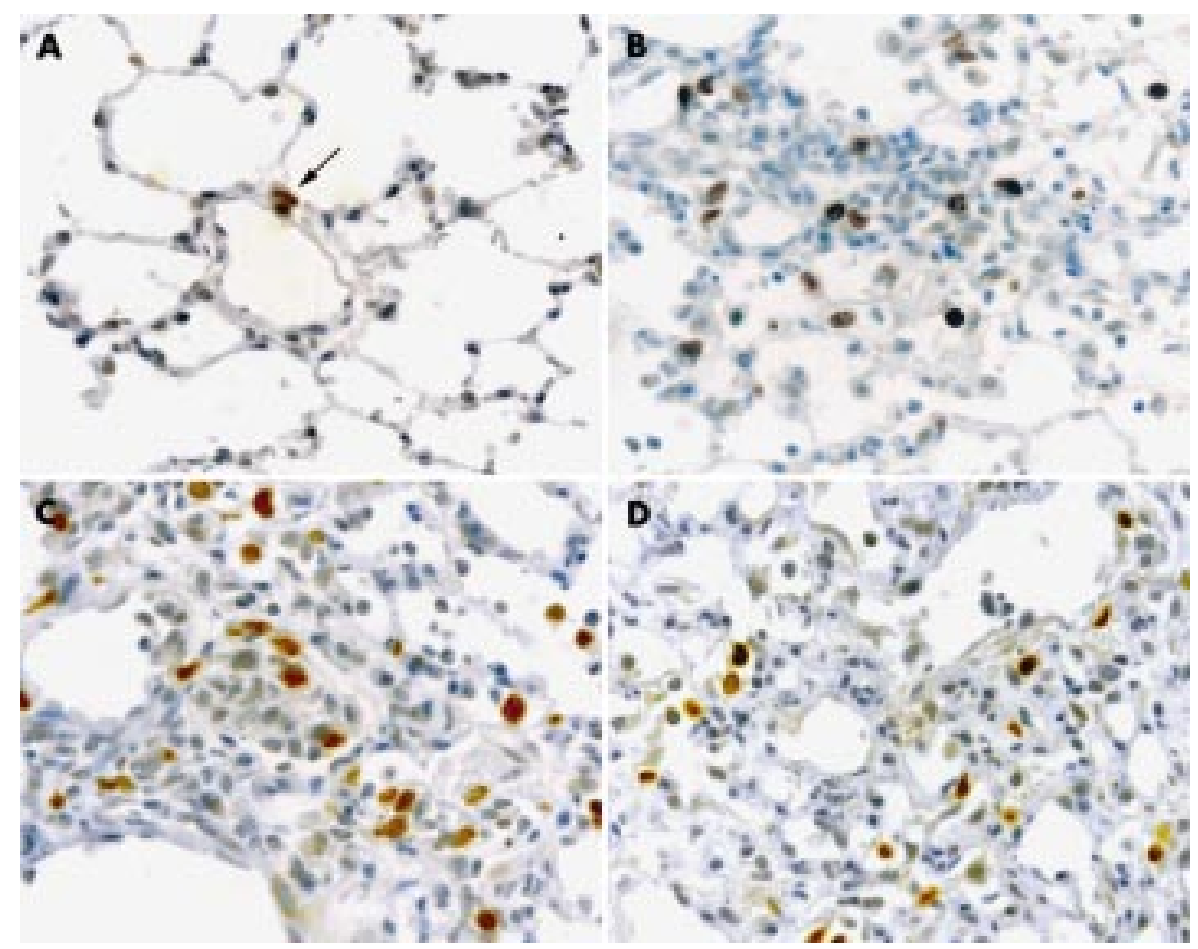

Figure 4 BrdU immunoreactive cells in (A) control lung tissue 3 days after saline instillation, (B) 3 days after bleomycin instillation, (C) 5 days after bleomycin instillation without dexamethasone treatment, and (D) 5 days after bleomycin instillation with dexamethasone. Magnification $\times 400$.

unable to reduce TGF- $\beta_{1}$ levels in vivo is supported by in vitro findings that dexamethasone did not reduce TGF- $\beta$ secretion by alveolar macrophages which had previously been activated in vivo by bleomycin. ${ }^{35}$

BAL fluid from bleomycin treated animals has been shown to contain mitogenic activity for lung fibroblasts, due partly to PDGF and thrombin. ${ }^{19} 20$ In addition, in vivo inhibition of thrombin and PDGF activity reduced bleomycin induced pulmonary fibrosis in rats. ${ }^{39}$ The mitogenic activity of BAL fluid

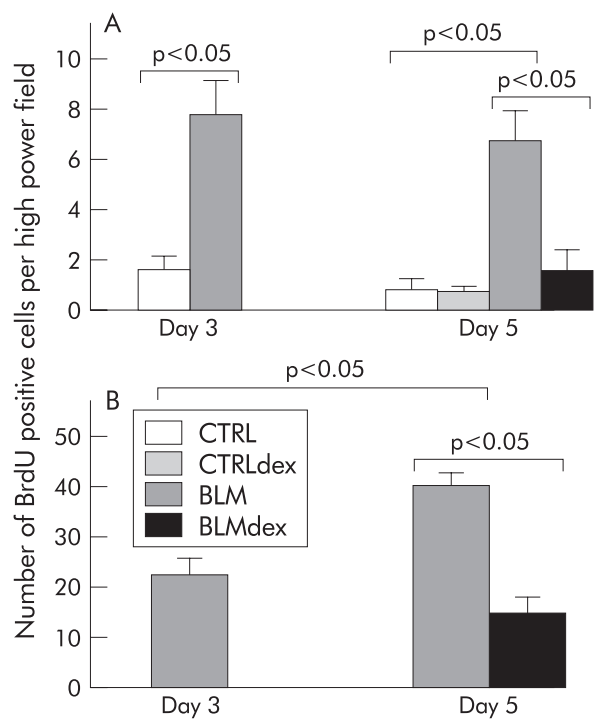

Figure 5 Number of BrdU immunoreactive cells presented as mean number of positive cells of 10 high power fields at $400 x$ magnification in $(A)$ areas of normal appearance lung tissue 3 and 5 days after bleomycin or saline instillation with and without dexamethasone treatment and (B) bleomycin injured lung tissue 3 days after bleomycin instillation and 5 days after bleomycin instillation with and without dexamethasone treatment. was significantly increased 3 days after bleomycin treatment and returned to control levels by day 7 , which supports previously reported findings. ${ }^{19}$ Treatment with dexamethasone had no effect on the mitogenic activity of BAL fluid in animals exposed to bleomycin. Both PDGF-AB levels and thrombin activity were significantly increased at day 3 after bleomycin, and presumably contributed to BAL fluid mitogenicity at that time point. Thrombin activity decreased gradually thereafter but remained significantly higher than in controls. The PDGF-AB levels remained increased at a relatively constant level during the whole study period, suggesting that the main source of PDGF was not inflammatory cells but was more likely to be resident cells. Thrombin activity and PDGF-AB levels in BAL fluid were not modulated by dexamethasone treatment of animals exposed to bleomycin. These data suggest that, although a 3 day course of dexamethasone is able to inhibit bleomycin induced pulmonary fibrosis in rats, this is not achieved by reducing levels of the fibrogenic mediators TGF- $\beta_{1}$, PDGF-AB, or thrombin in BAL fluid.

We chose to examine TGF- $\beta_{1}$, PDGF, and thrombin levels as these are among the most important fibrogenic mediators known to play a role in the pathophysiology of pulmonary fibrosis. ${ }^{1348934}$ However, other mediators such as endothelin-1, insulin-like growth factor-1, interleukin (IL)11, IL-13, and members of the fibroblast and epidermal growth factor families may also be involved. ${ }^{6{ }^{36-38}}$ From our study we cannot exclude the possibility that treatment with dexamethasone exerts its antifibrotic effect via mechanisms involving mediators other than TGF- $\beta_{1}$, PDGF, and thrombin. We also cannot rule out the possibility that dexamethasone treatment affects the parenchymal levels or activity of these growth factors. For instance, fibroblasts have also been identified as a source of TGF- $\beta_{1}$ in pulmonary fibrosis, ${ }^{39}$ and it has been shown that dexamethasone reduces TGF- $\beta_{1}$ mRNA and TGF- $\beta$ secretion by lung fibroblasts. ${ }^{40}$ In addition, dexamethasone is known to reduce collagen synthesis and steady state levels of procollagen mRNA by lung fibroblasts. ${ }^{41}$ The dexamethasone regimen used in our study may therefore have reduced parenchymal TGF- $\beta_{1}$ expression by fibroblasts with 
subsequent decreased autocrine stimulation of collagen synthesis. Alternatively, stimulation of fibroblasts with dexamethasone stimulates the production of the proteoglycan decorin that binds and inactivates TGF- $\beta .^{42}$ Transient expression of decorin in the lungs from bleomycin treated animals has been shown to reduce pulmonary fibrosis without reducing total TGF- $\beta_{1}$ levels in BAL fluid. ${ }^{32}$ It is therefore possible that the dexamethasone treatment in our study reduced parenchymal TGF- $\beta_{1}$ activity by increasing the decorin levels in the lung with decreased collagen deposition as a consequence.

Intratracheal instillation of bleomycin resulted in BrdU incorporation into cells resembling alveolar type II epithelial cells in areas of normal lung architecture and in bleomycin injured areas, indicating an increase in the number of proliferating cells. This finding supports previous studies and shows that active epithelial proliferation contributes to repair of the injured epithelial surface within the first few days of injury ${ }^{43}{ }^{44}$ In this study we examined cell proliferation in lung tissue from bleomycin treated and control animals after 3 and 5 days. Later time points were not examined as previous studies have shown that maximal cell proliferation occurs within the first week after bleomycin exposure. ${ }^{20}{ }^{44}$ Treatment with dexamethasone reduced the number of BrdU stained cells in both injured and normal areas of the lung parenchyma in bleomycin treated animals. This suggests that dexamethasone either prevents or delays cell proliferation. Apoptosis is also known to play a key role in the development of bleomycin induced pulmonary fibrosis. ${ }^{45}$ In addition, it has been reported that prolonged administration of a high dose of methylprednisolone $(100 \mathrm{mg} / \mathrm{kg} / \mathrm{day})$ suppressed bronchial and alveolar epithelial apoptosis and reduced evidence of histological fibrosis induced by bleomycin. ${ }^{46}$ Dexamethasone may therefore have also reduced epithelial cell apoptosis which, together with reduced fibroblast proliferation, prevented or delayed the development of fibrosis. However, TUNEL staining for apoptotic cells on lung sections from the current study were inconclusive (data not shown).

This study is the first to show that a 3 day course of treatment with dexamethasone, started after initiation of bleomycin induced fibrosis and given during the major proliferative phase, is able to reduce excessive pulmonary collagen deposition in rats. The observed effect of dexamethasone is established without a reduction in inflammatory cell numbers or the fibrogenic mediators TGF- $\beta_{1}$, PDGF-AB, and thrombin in the alveolar space. We speculate that the antifibrotic effect of dexamethasone is mediated via other pathways which may include reduced or delayed cell proliferation, decreased epithelial apoptosis, or inhibition of tissue profibrotic mediator activity. Our findings are of important clinical relevance as corticosteroid treatment is the mainstay of treatment in patients with pulmonary fibrosis, ARDS, and neonatal respiratory distress syndrome, and the timing of initiating treatment appears to be crucial for its antifibrotic effects.

\section{ACKNOWLEDGEMENTS}

This work was supported by a grant from the Royal Dutch Academy of Sciences.

\section{Authors' affiliations}

W A Dik, M A Versnel, B A E Naber, Department of Immunology, Division of Neonatology, Erasmus MC, University Medical Centre, Rotterdam, The Netherlands

B A E Naber, L J I Zimmermann, Department of Pediatrics, Division of Neonatology, Erasmus MC, University Medical Centre, Rotterdam, The Netherlands

R J McAnulty, G J Laurent, S E Mutsaers, Centre for Cardiopulmonary Biochemistry and Respiratory Medicine, University College London

Medical School, Rayne Institute, London, UK

\section{REFERENCES}

1 McAnulty RJ, Laurent GJ. Pathogenesis of lung fibrosis and potential new therapeutic strategies. Exp Nephrol 1995;3:96-107.
2 Coker RK, Laurent GJ. Anticytokine approaches in pulmonary fibrosis: bringing factors into focus. Thorax 1997;52:294-6.

3 Yoshida M, Sakuma-Mochizuki J, Abe K, et al. In vivo gene transfer of an extracellular domain of platelet-derived growth factor beta receptor by the HVJ-liposome method ameliorates bleomycin-induced pulmonary fibrosis. Biochem Biophys Res Commun 1999:265:503-8.

4 Khalil N, Bereznay O, Sporn M, et al. Macrophage production of transforming growth factor beta and fibroblast collagen synthesis in chronic pulmonary inflammation. J Exp Med 1989;170:727-37.

5 Maeda A, Hiyama K, Yamakido $H$, et al. Increased expression of platelet-derived growth factor $\mathrm{A}$ and insulin- like growth factor-l in BAL cells during the development of bleomycin- induced pulmonary fibrosis in mice. Chest 1996;109:780-6.

6 Mutsaers SE, Foster ML, Chambers RC, et al. Increased endothelinand its localization during the development of bleomycin-induced pulmonary fibrosis in rats. Am J Respir Cell Mol Biol 1998;18:611-9.

7 Hernnas J, Nettelbladt O, Bjermer L, et al. Alveolar accumulation of fibronectin and hyaluronan precedes bleomycin- induced pulmonary fibrosis in the rat. Eur Respir J 1992;5:404-10.

8 Hernandez-Rodriguez NA, Cambrey AD, Harrison NK, et al. Role of thrombin in pulmonary fibrosis. Lancet 1995;346:1071-3.

9 Howell DC, Goldsack NR, Marshall RP, et al. Direct thrombin inhibition reduces lung collagen, accumulation, and connective tissue growth factor mRNA levels in bleomycin-induced pulmonary fibrosis. Am J Pathol 2001; 159:1383-95.

10 Marshall R, Bellingan $G$, Laurent $G$. The acute respiratory distress syndrome: fibrosis in the fast lane. Thorax 1998;53:815-7.

11 Marshall RP, Bellingan G, Webb S, et al. Fibroproliferation occurs early in the acute respiratory distress syndrome and impacts on outcome. Am Respir Crit Care Med 2000;162:1783-8.

12 Meduri GU, Chinn AJ, Leeper KV, et al. Corticosteroid rescue treatment of progressive fibroproliferation in late ARDS. Patterns of response and predictors of outcome. Chest 1994;105:1516-27.

13 Meduri GU, Tolley EA, Chinn A, et al. Procollagen types I and III aminoterminal propeptide levels during acute respiratory distress syndrome and in response to methylprednisolone treatment. Am J Respir Crit Care Med 1998;158:1432-41.

14 Anderson WR, Engel RR. Cardiopulmonary sequelae of reparative stages of bronchopulmonary dysplasia. Arch Pathol Lab Med 1983;107:603-8

15 Sanders RJ, Cox C, Phelps DL, et al. Two doses of early intravenous dexamethasone for the prevention of bronchopulmonary dysplasia in babies with respiratory distress syndrome. Pediatr Res 1994;36:122-8.

16 Garland JS, Alex CP, Pauly TH, et al. A three-day course of dexamethasone therapy to prevent chronic lung disease in ventilated neonates: a randomized trial. Pediatrics 1999;104:91-9.

17 Lazenby AJ, Crouch EC, McDonald JA, et al. Remodeling of the lung in bleomycin-induced pulmonary fibrosis in the rat. An immunohistochemical study of laminin, type IV collagen, and fibronectin. Am Rev Respir Dis 1990; 142:206-14.

18 Laurent GJ, McAnulty RJ. Protein metabolism during bleomycin-induced pulmonary fibrosis in rabbits. In vivo evidence for collagen accumulation because of increased synthesis and decreased degradation of the newly synthesized collagen. Am Rev Respir Dis 1983;128:82-8

19 Walsh J, Absher M, Kelley J. Variable expression of platelet-derived growth factor family proteins in acute lung injury. Am J Respir Cell Mo Biol 1993;9:637-44

20 Tani K, Yasuoka S, Ogushi F, et al. Thrombin enhances lung fibroblast proliferation in bleomycin-induced pulmonary fibrosis. Am J Respir Cell Mol Biol 1991;5:34-40

21 Phan SH, Thrall RS, Williams C. Bleomycin-induced pulmonary fibrosis. Effects of steroid on lung collagen metabolism. Am Rev Respir Dis $1981 ; 124: 428-34$

22 Koenig WJ, Cross CE, Hesterberg TW, et al. The smoking gun. Mechanism of methylprednisolone prevention of bleomycin-induced pulmonary fibrosis. Chest 1983;83:5-7S

23 Grunze MF, Parkinson D, Sulavik SB, et al. Effect of corticosteroids on lung volume-pressure curves in bleomycin- induced lung injury in the rat. Exp Lung Res 1988;14:183-95.

24 Laurent GJ, Cockerill P, McAnulty RJ, et al. A simplified method for quantitation of the relative amounts of type I and type III collagen in small tissue samples. Anal Biochem 1981;113:301-12

25 Oliver MH, Harrison NK, Bishop JE, et al. A rapid and convenient assay for counting cells cultured in microwell plates: application for assessment of growth factors. J Cell Sci 1989:92:513-8.

26 Lottenberg R, Christensen U, Jackson CM, et al. Assay of coagulation proteases using peptide chromogenic and fluorogenic substrates. Methods Enzymol 1981;80:341-61

27 Dik WA, Zimmermann LJ, Naber BA, et al. Thrombin contributes to bronchoalveolar lavage fluid mitogenicity in lung disease of the premature infant. Pediatr Pulmonol 2003:35:34-41.

28 Fine A, Goldstein RH. The effect of transforming growth factor-beta on cell proliferation and collagen formation by lung fibroblasts. J Biol Chem 1987;262:3897-902

29 Hoyt DG, Lazo JS. Alterations in pulmonary mRNA encoding procollagens, fibronectin and transforming growth factor-beta precede bleomycin-induced pulmonary fibrosis in mice. J Pharmacol Exp Ther 1988;246:765-71.

30 Giri SN, Hyde DM, Hollinger MA. Effect of antibody to transforming growth factor beta on bleomycin induced accumulation of lung collagen in mice. Thorax 1993;48:959-66.

31 Wang Q, Wang Y, Hyde DM, et al. Reduction of bleomycin induced lung fibrosis by transforming growth factor beta soluble receptor in hamsters. Thorax 1999;54:805-12. 
32 Kolb M, Margetts PJ, Galt T, et al. Transient transgene expression of decorin in the lung reduces the fibrotic response to bleomycin. Am J Respir Crit Care Med 2001;163:770-7.

33 Yi ES, Salgado M, Williams S, et al. Keratinocyte growth factor decreases pulmonary edema, transforming growth factor-beta and platelet-derived growth factor-BB expression, and alveolar type II cell loss in bleomycin-induced lung injury. Inflammation 1998;22:315-25.

34 Coker RK, Laurent GJ, Shahzeidi S, et al. Transforming growth factors-beta 1, -beta 2, and -beta 3 stimulate fibroblast procollagen production in vitro but are differentially expressed during bleomycin-induced lung fibrosis. Am J Pathol 1997;150:981-91.

35 Khalil N, Whitman C, Zuo L, et al. Regulation of alveolar macrophage transforming growth factor-beta secretion by corticosteroids in bleomycin-induced pulmonary inflammation in the rat. J Clin Invest 1993:92:1812-8.

36 Mutsaers SE, Bishop JE, McGrouther G, et al. Mechanisms of tissue repair: from wound healing to fibrosis. Int J Biochem Cell Biol 1997;29:5-17.

37 Zhu Z, Lee CG, Zheng T, et al. Airway inflammation and remodeling in asthma. Lessons from interleukin 11 and interleukin 13 transgenic mice. Am J Respir Crit Care Med 2001;164:S67-70.

38 Belperio JA, Dy M, Burdick MD, et al. Interaction of IL-13 and C10 in the pathogenesis of bleomycin-induced pulmonary fibrosis. Am J Respir Cell Mol Biol 2002;27:419-27.
39 Santana A, Saxena B, Noble NA, et al. Increased expression of transforming growth factor beta isoforms (beta 1, beta 2, beta 3 ) in bleomycin-induced pulmonary fibrosis. Am J Respir Cell Mol Bio $1995 ; 13: 34-44$

40 Shull' S, Meisler N, Absher M, et al. Glucocorticoid-induced down regulation of transforming growth factor- beta 1 in adult rat lung fibroblasts. Lung 1995;173:71-8.

41 Sterling KM, Jr., Harris M, Mitchell JJ, et al. Dexamethasone decreases the amounts of type I procollagen mRNAs in vivo and in fibroblast cell cultures. J Biol Chem 1983;258:7644-7.

42 Kahari VM. Hakkinen L, Westermarck J, et al. Differential regulation of decorin and biglycan gene expression by dexamethasone and retinoic acid in cultured human skin fibroblasts. J Invest Dermatol 1995; 104:503-8.

43 Adamson IY, Young L, Bowden DH. Relationship of alveolar epithelial injury and repair to the induction of pulmonary fibrosis. Am J Pathol 1988:130:377-83

44 Khalil N, O'Connor RN, Flanders KC, et al. Regulation of type II alveolar epithelial cell proliferation by TGF-beta during bleomycin-induced lung injury in rats. Am J Physiol 1994;267:L498507

45 Kuwano K, Hagimoto N, Tanaka T, et al. Expression of apoptosis-regulatory genes in epithelial cells in pulmonary fibrosis in mice. J Pathol 2000;190:221-9.

46 Hagimoto N, Kuwano K, Nomoto Y, et al. Apoptosis and expression of Fas/Fas ligand mRNA in bleomycin-induced pulmonary fibrosis in mice. Am J Respir Cell Mol Biol 1997; 16:91-101.

\section{LUNG ALERT}

Radiofrequency ablation of lung cancer

$\Delta$ Herrera L, Fernando HC, Perry Y, et al. Radiofrequency ablation of pulmonary malignant tumors in nonsurgical candidates. J Thorac Cardiovasc Surg 2003;125:929-37

ung cancer is the most common cause of cancer related death in the USA. Surgical resec-

tion is the treatment of choice in non-small cell lung cancer (NSCLC). Many patients have

- poor pulmonary function or other medical co-morbidities and are not suitable for resection. Such patients are offered alternative treatments such as radiotherapy and chemotherapy, with poorer long term survival. Radiofrequency ablation (RFA) is a thermal energy delivery system that applies alternating current through a needle electrode into the tumour. It has been used for treating unresectable liver tumours.

In this pilot study at the University of Pittsburgh, 33 tumour nodules in 18 patients were treated with RFA. Five of the patients had NSCLC (stage I $(n=3)$, stage IV $(n=2)$ ) and 13 had pulmonary metastases from an extrathoracic malignancy (sarcoma, colon, melanoma, renal). Two of the five patients with NSCLC had recurrence of disease after thoracotomy and resection. All patients underwent a chest CT scan before and after RFA. Patients were followed up at 1 and 3 month intervals with a CT scan of the chest. At a median follow up of 6 months (range 1-10) four of the five patients with NSCLC were alive; the other patient died at the age of 95 years without evidence of disease progression. Seven of the 13 patients with pulmonary metastases were alive at a median follow up of 4 months. The response rate was better for lesions less than $5 \mathrm{~cm}$ in diameter.

Although RFA will not replace surgical resection as the treatment of choice for NSCLC, it may offer better survival in selected patients and additional trials are needed to determine its role.

P Bhatio

Specialist Registrar, Respiratory/GIM Blackpool Victoria Hospital, Blackpool, UK Naviapo@aol.com 